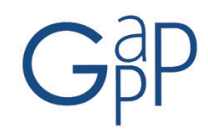

GESTIÓN Y ANÁLISIS DE POLÍTICAS PÚBLICAS, Nueva Época, n 10 julio-diciembre 2013 ISSN: 1989-8991

DOI: http://dx.doi.org/10.24965/gapp.voi10.10115

\title{
La evaluación del rendimiento individual. Un instrumento válido para lograr la eficiencia en la gestión de Recursos Humanos en las Administraciones Públicas
}

\author{
José María Pablos Teijeiro \\ Universidad de Cádiz \\ josemaria.pablos@uca.es \\ José María Biedma Ferrer \\ Universidad de Cádiz \\ josemaria.biedma@uca.es \\ Recibido: 16 de mayo de 2013 \\ Aceptado: 27 de noviembre de 2013
}

\begin{abstract}
Resumen
En el contexto de crisis económica que padece nuestra sociedad, el presente artículo aborda la necesidad y conveniencia de implantar en la gestión de los recursos humanos del sector público técnicas de evaluación del rendimiento individual, con el fin de lograr mayor eficiencia. Tras un estudio de la herramienta de evaluación del rendimiento en la empresa privada, el análisis profundiza en las posibilidades y limitaciones que la implementación de dicha técnica tiene en el sector público, con detenimiento en la referencia que el Estatuto Básico del Empleado Público realiza a la evaluación del rendimiento. Para finalizar se sugieren líneas futuras de investigación en este terreno.
\end{abstract}

Palabras clave

Evaluación del rendimiento, gestión del rendimiento, gestión pública, recursos humanos, EBEP, desempeño

\section{Individual Performance Evaluation. A Valid Instrument for Achieving Efficiency in Human Resources Management Goverment}

\section{Abstract}

In the context of economic crisis in our society, this paper discusses the need and desirability of implementing in the management of public sector human resources performance evaluation techniques individually, in order to achieve greater efficiency. After a study of the performance evaluation tool in the private sector, the analysis explores the possibilities and limitations that the implementation of this technique in the public sector, with reference length in the Civil Service Basic Statute makes the performance evaluation. Finally we suggest future research in this area.

\section{Keywords}

Performance appraisal, performance management, public administration, human resources, EBEP, performance 


\section{INTRODUCCIÓN}

Actualmente cualquier responsable de la gestión de las organizaciones es consciente de la importancia de los recursos humanos en el éxito y desarrollo de las mismas, para mantener la competitividad en un entorno dinámico e incierto (Dolan, 2007; De la Calle y Ortiz, 2004). Como es sabido, los factores que están ligados a la competitividad son aquellos que dependen de los recursos humanos, de sus habilidades, destrezas, competencias, etc. Ello ha motivado el interés creciente de los investigadores por la función empresarial relacionada con la gestión de personas. Siguiendo a Valle-Cabrera (2003), podemos decir que la transformación experimentada por la función de personal desde sus inicios ha supuesto que la dirección de las organizaciones haya ido considerando cada vez más a los recursos humanos como factor determinante de su éxito o fracaso.

El hecho de que los recursos humanos constituyan uno de los principales factores de éxito competitivo en las empresas es ampliamente aceptado por la literatura (entre otros, Clifford y Cavanagh, 1985; Huck y McEwen, 1991; Pfeffer, 1994; Yusuf, 1995; Luck, 1996; Camisón, 1997; Monfort, 2000; Warren y Hutchinson, 2000).

Los procesos que tradicionalmente han constituido la gestión de recursos humanos (Ferris, et al., 1999; Longo, 2005; Dolan et al., 2007) son los siguientes:

- Procesos básicos: planificación y análisis del puesto de trabajo.

- Procesos de afectación: reclutamiento, selección, socialización y procesos sustractivos.

- Procesos de desarrollo: formación y gestión de carrera profesional.

- Procesos de desempeño: evaluación del rendimiento.

- Procesos de compensación: retribución y compensaciones.

El planteamiento, por tanto, es maximizar la efectividad de las prácticas de recursos humanos dentro de la organización contando con unos responsables que sepan cuáles de las anteriores considerar y cómo implantarlas. Para ello la capacidad técnica es fundamental.

La gestión de la evaluación del rendimiento como proceso de recursos humanos, y la forma de medirlo, suponen un tema de creciente importancia a nivel internacional.

Si se analizan las tendencias en la gestión de recursos humanos en las Administraciones Públicas de los países más desarrollados, nos podemos encontrar con la necesidad, cada vez más importante, de una correcta planificación estratégica de recursos humanos (Villoria, 2009). El objetivo de dicha planificación no es otro que el de poder disponer de las personas necesarias en cada momento para acometer las acciones oportunas que permitan implantar con éxito la estrategia (Valle-Cabrera, 2003). Esto incluiría tanto los procesos de reclutamiento y selección como los de reducción de personal. Igualmente, y como continuación a lo anterior, es necesario implementar estrategias de estímulo de competencias que permitan motivar al empleado público, para que desarrolle conductas adecuadas y se esfuerce en la consecución de los objetivos estratégicos, ligando este rendimiento a los sistemas de retribución salariales.

En este sentido resulta necesario plantearse la elaboración de políticas y líneas de actuación conducentes a la adecuación y mejora del empleo público. Entre otras, la actuación en los niveles de dirección de personal, la planificación general de los recursos humanos y su adecuación estratégica a los contextos organizativos cambiantes y a nivel administrativo en el desarrollo y plasmación de las medidas anteriores a la gestión cotidiana de personal (Olías de Lima, 2001).

El objetivo del presente trabajo de investigación es realizar un análisis de la situación actual en la investigación académica sobre la evaluación del rendimiento, para poder así detectar posibles líneas de investigación futuras que permitan determinar de qué forma es posible utilizar dicho instrumento de gestión de recursos humanos en las Administraciones Públicas, de cara a la mejora del rendimiento, la efectividad y la eficiencia de los trabajadores públicos.

Para ello, este documento se divide en cuatro secciones. La primera sección ofrece una visión general de los procesos de gestión de recursos humanos en la actualidad y su alineación con los objetivos estratégicos de las organizaciones, así como una revisión bibliográfica del concepto de evaluación del rendimiento y de los aspectos más relevantes de la gestión del rendimiento como proceso. En la sección segunda se analizan las transformaciones que están sufriendo las Administraciones para alcanzar los fines públicos con mayor eficiencia, eficacia y satisfacción del ciudadano y cómo la evaluación del rendimiento de los empleados públicos contribuye en ese sentido. En la sección tercera se hace una revisión de la literatura correspondiente al Estatuto Básico del Empleado Público 
(EBEP) y su relación con los procesos de evaluación de los empleados públicos. Por último, en la sección cuarta, se presentan las conclusiones, una propuesta de líneas de investigación así como las limitaciones inherentes a los procesos de evaluación del rendimiento en las Administraciones Públicas.

\section{LA EVALUACIÓN DEL DESEMPEÑO EN LAS INSTITUCIONES PRIVADAS. LA GESTIÓN ESTRATÉGICA DE LOS RECURSOS HUMANOS}

El modo de gestionar los recursos humanos en las organizaciones ha evolucionado a lo largo del tiempo (Ferris et al., 1999). Desde las concepciones más “administrativas" de la función de los recursos humanos (en adelante, RRHR) se ha evolucionado a una visión proactiva de la misma (Valle-Cabrera, 2003; Leal, 2004), más en la línea de adecuar la gestión de personas con los objetivos estratégicos generales de la organización (Ferris et al., 1999).

La consideración de la relación estrategia-dirección de recursos humanos implica que el Recurso Humano es un valioso activo y un factor potencial de ventaja competitiva.

Siguiendo a Peteraf y Barney (2003: 314), podemos considerar que una empresa posee ventaja competitiva si "es capaz de crear más valor económico que su competidor marginal en su mismo mercado de productos". Según ésta definición, las organizaciones que pretendan obtener rendimientos superiores a los de sus competidores deberán "encontrar" aquel factor o factores que les permita mantener esa ventaja en el tiempo.

En este punto, y desde la perspectiva del enfoque de recursos y capacidades, podemos decir que la fuente principal de ventaja competitiva para la organización se encuentra en sus recursos y en la forma en que éstos se coordinan e interrelacionan, es decir, en sus capacidades. La capacidad de los RRHH para proporcionar ventaja competitiva ha sido analizada por varios autores (Barney, 1991; Grant, 1991 y Barney y Delwyn, 2007). En este sentido cabe destacar el estudio de Barney y Wright (1998). Estos autores analizan qué prácticas de RRHH son simplemente generadoras de valor y qué requisitos deben cumplir para llegar a ser una verdadera fuente de ventaja competitiva. El reto, por tanto, es el de implementar sistemas de procesos de recursos humanos que creen un efecto sinérgico, frente al desarrollo independiente de "buenas prácticas" de recursos humanos. (Barney y Wright 1998: 40).

Si se parte de lo indicado anteriormente, podemos llegar a la conclusión de que el factor humano puede constituir una de las fuentes de ventaja competitiva sostenible más importante para las organizaciones (Valle-Cabrera, 2003: 41).

También cabe señalar que la alineación de los procesos de RRHH con los objetivos globales (estratégicos) de las organizaciones ocasiona un campo de investigación como es el de la gestión estratégica de los RRHH (Ferris et al., 1999). Ello supone la existencia de una fuerte interdependencia y coordinación entre las diferentes políticas y actividades funcionales de RRHH, y de todas las prácticas de RRHH con la estrategia de la organización, respectivamente (Albizu y Lertxundi, 2011: 282).

Dentro de esta perspectiva estratégica de la gestión de recursos humanos Delaney et al. (1989), Huselid (1995), Delery and Doty (1996), citados en Ferris et al. (1999) y Valle-Cabrera (2003), destacan las "estrategias de valoración del personal", es decir, la forma en que las personas alcanzarán mediante sus conductas los resultados esperados. Para ello es necesario proceder del siguiente modo: en primer lugar, fijar unos objetivos operativos claros; y en segundo lugar, establecer un sistema de recompensas que esté ligado a los rendimientos de las personas o a algún otro tipo de resultados de la organización.

Para implantar o mantener con éxito la estrategia empresarial, la evaluación del rendimiento supone un elemento clave. La evaluación del desempeño ha ido aumentando su importancia como práctica de recursos humanos debido al incremento de las exigencias de innovación, de la competencia y por las repercusiones de la tecnología en la naturaleza de los puestos de trabajo (Dolan, 2007). La importancia de dicha evaluación se debe a que ésta determina la orientación de los comportamientos y de los resultados buscados. Por tanto, es de suma importancia en las organizaciones actuales la implantación y adaptación de sistemas de evaluación del desempeño de sus empleados para valorar los comportamientos necesarios con el fin de lograr los objetivos estratégicos.

\section{Aproximación al concepto de evaluación del desempeño}

La valoración del rendimiento del personal supone uno de los procesos básicos, complejos y que plantea más controversias a los gestores de recursos humanos (Badawy, 1988; Roberts, 2002). Se trata de una responsabilidad y actividad fundamental de la dirección. Por ello, toda organización debería contar con algún sistema de valoración del rendimiento que le permita obtener información sobre la contribución de todos y cada uno de los empleados a la consecución de los objetivos establecidos. Numerosas encuestas y estudios realizados reciente- 
mente destacan la importancia de la gestión del rendimiento en las organizaciones, tanto públicas como privadas. No obstante, sólo un tercio de los trabajadores creen que estos sistemas les ayudan a mejorar su rendimiento (Aguinis et al., 2011).

Las funciones y tareas que desarrolla un trabajador tienen como objetivo la obtención de un determinado resultado. El rendimiento laboral, su medida, exigencia y mejora deben formar parte, por tanto, del sistema de evaluación del personal (García-Tenorio, 2005: 190). La evaluación del rendimiento es en sí misma un compromiso entre empleado y organización. Si el empleado desarrolla sus conocimientos y competencias en la búsqueda de los mejores resultados, la organización, como contrapartida, le aportará reconocimiento, oportunidades de formación y desarrollo e incentivos. Por ello parece necesario que el empleado participe en el proceso de evaluación para que el sistema de evaluación sea realmente efectivo (Roberts, 2002).

Un sistema de evaluación del rendimiento efectivo tiene dos objetivos principales: por un lado, generar información sobre la cual se tomaran decisiones en materia de salarios y promoción; y, en segundo lugar, identificar aquellas áreas que serán susceptibles de futuros procesos de desarrollo y/o crecimiento (Badawy, 2007).

La evaluación del desempeño (en adelante, EDD) implica la identificación, medida y gestión del rendimiento de las personas de una organización (Carroll y Schneir, 1982). La identificación significa determinar las áreas de trabajo que van a ser susceptibles de evaluación y que afectan al éxito de la organización. Dado que evaluar implica la realización de un juicio sobre el correcto o incorrecto rendimiento del empleado, el punto central del sistema de evaluación es la medición. También cabe señalar que gestionar supone el objetivo central del sistema de evaluación. La evaluación del desempeño obliga a aportar a los trabajadores una referencia sobre cual, o cuáles, debería/n ser su objetivo/s, en términos de rendimiento, para alcanzar su máximo potencial en la organización.

Para Leal (2004) la valoración del rendimiento, cómo técnica de gestión de recursos humanos, se orienta a recabar información sobre la actuación de cada una de las personas de la organización, en relación a una serie de factores establecidos de forma previa, con el fin de determinar cuál ha sido su aportación a la consecución de los objetivos individuales, departamentales y globales. En este sentido, se ha definido la evaluación del desempeño como "un procedimiento continuo, sistemático, orgánico y en cascada, de expresión de juicios acerca del personal de una empresa, en relación con su trabajo habitual, que pretende sustituir a los juicios ocasionales y formulados de acuerdo con los más variados criterios" (Puchol, 1997: 308).

Otros autores (Dolan et al., 2007) incorporan a la definición de la EDD aspectos novedosos como la productividad. La mejora de la productividad es una cuestión recurrente en las organizaciones. La productividad relaciona la cantidad de output obtenido por unidad de factor (mano de obra) utilizada. Ciertamente, las mejoras en productividad son debidas al uso de innovaciones tecnológicas, organizativas o humanas, aunque también es posible incrementarlas a partir de mejoras en el rendimiento de los recursos humanos. De esta forma la evaluación del rendimiento se define como un procedimiento estructural y sistemático para medir, evaluar e influir sobre los atributos, comportamientos y resultados relacionados con el trabajo, con el fin de descubrir en qué medida es productivo el empleado, y si podrá mejorar su rendimiento futuro (Dolan et al., 2007: 229).

Como podemos observar en las anteriores definiciones hay una cierta diversidad terminológica. Así, encontramos términos como rendimiento, resultados o desempeño. En otros casos se refieren a ella utilizando la siguiente terminología: valoración, evaluación o calificación del personal, valoración del mérito o de resultados.

Es posible establecer, por tanto, una diferencia entre "gestión del desempeño" (performance management) entendiendo éste como el "proceso continuo de identificación, medición y evolución del rendimiento de individuos y equipos y su ajuste con los objetivos estratégicos de rendimiento de la organización” y "evaluación del rendimiento" (performance appraisal), como "el proceso administrativo del área de recursos humanos consistente en representar las fortalezas y debilidades de los empleados de una organización en un periodo de tiempo generalmente un año" (Aguinis, 2009:2).

En definitiva, la EDD es una actividad fundamental para la empresa. El desafío (García-Tenorio, 2005: 193) consiste en gestionar el sistema de evaluación de tal forma que permita conseguir los objetivos de mejora del rendimiento y desarrollo de los empleados. En el futuro la prueba que habrá que superar reside en la capacidad de las organizaciones de ir más allá de la evaluación del rendimiento, hasta alcanzar lo que se ha denominado "gestión del rendimiento".

\section{El sistema de evaluación del desempeño}

Para establecer un sistema de evaluación del desempeño, es necesario tomar una serie de decisiones respecto a qué medir, quién debe realizar las valoraciones, cómo efectuar las mediciones, cuándo deben efectuarse y en 
qué contexto deberán tener lugar. Igualmente se deben de tener en cuenta los aspectos claves de lo que hemos definido como evaluación del rendimiento o evaluación del desempeño.

La mayoría de las organizaciones disponen de alguna forma de evaluar el desempeño individual. Sin embargo, pocas de ellas pueden asegurar que el sistema de evaluación que utilizan les permita obtener los mejores resultados a lo largo del tiempo. Los estudios previos realizados por Martín y Bartol (1998: 223) sostienen que las acciones necesarias para el mantenimiento de un sistema de EDD pueden dividirse en tres categorías: gestionar el sistema, controlar el sistema e implementar feedbacks de información para aquellos que utilizan el sistema. Siguiendo esta línea de estudio se encuentra Longo (2005:134), quien describe el ciclo de la gestión del rendimiento como un proceso que se inicia con la planificación y seguimiento del mismo, originando la evaluación un proceso de feedback que re-inicia el proceso. En la misma línea, Aguinis (2009) añade que lo anteriormente expuesto no podría llevarse a cabo si antes la organización no diseña el "marco" en el que se debe realizar la evaluación.

Los comienzos de todo proceso de evaluación en la organización deben consistir en el diseño de un sistema ideal que desarrolle todo el proceso de EDD. No obstante, con posterioridad será el día a día el que determinará la validez de dicho sistema y sus posibles modificaciones.

Un sistema de EDD ideal debe recoger aquellos aspectos de la tarea que sean relevantes para la identificación de cuáles son los resultados esperados por el departamento de recursos humanos. El estudio realizado por Brown et al. (2010) relaciona de forma positiva el compromiso del empleado con su organización, la satisfacción laboral y el deseo de permanecer en la organización con la calidad del proceso de evaluación del desempeño. Las consecuencias de esta relación pueden verse reflejados en mejoras de la productividad y eficiencia, así como en la efectividad de las tareas. Por tanto, el papel de un sistema de evaluación de calidad debe basarse en estimular el rendimiento del empleado (Aguinis, 2009), siendo la figura del evaluador sumamente relevante en todo proceso de EDD.

Leal (2004: 362), citando a Claver, Gascó y Llopis (1995), establece que "el principal responsable o protagonista de este proceso ha de ser el directivo de línea pues es el que mejor situado se encuentra para realizar la evaluación”. En esta línea, diversos autores (Bowman, 1999; García-Tenorio, 2005; Dolan et al., 2007; Gómez-Mejía et al. 2008; Dessler, 2009) coinciden en la necesidad de que sea el supervisor directo del empleado quien evalúe su desempeño. No obstante, existen otras opciones alternativas novedosas. Así por ejemplo, basándose en la creencia de que el empleado tiene un conocimiento importante sobre la ejecución del trabajo, la autoevaluación puede aportar datos valiosos, especialmente cuando los objetivos globales se fijan de forma conjunta entre supervisor y empleado (Bowman, 1999). Sin embargo, este sistema adolece de fiabilidad, pues en algunos casos el empleado se asigna puntuaciones más altas de las que le corresponderían si fuera evaluado por otra persona. Otras alternativas que se utilizan como indicadores de rendimiento son las siguientes: la evaluación de los pares, las evaluaciones de equipo o grupo de empleados, la de equipos de trabajo auto-administrados o auto-dirigidos, y, por último, la evaluación de feedback o "evaluación 360 grados". Este último método engloba a los anteriores en el sentido de que el empleado pueda ser evaluado desde perspectivas distintas (compañeros, supervisor directo, clientes, otros supervisores... ) y reciba una retroalimentación productiva acerca de su rendimiento que le permita mejorar en su desarrollo profesional.

Otro aspecto a tener en consideración en todo proceso de EDD, como se ha apuntado anteriormente, es el de determinar qué es lo que se va a medir. Siguiendo a García-Tenorio (2005), podemos establecer tres tipos básicos de evaluación: evaluación de resultados, evaluación de la actuación y evaluación del potencial.

Con respecto a la evaluación de resultados (outcomes-based performance), cabe afirmar que ésta se identifica con el producto de la actuación en dicho trabajo o con la eficacia alcanzada en el mismo (Williams, 2003). La evaluación de resultados tiene su origen en los sistemas de dirección por objetivos. Los objetivos representan los fines que se persiguen por medio de una actividad de una u otra índole (Koontz, 1998). Por tanto, la administración por objetivos consiste en gestionar el cumplimiento eficaz y eficiente de los objetivos organizacionales e individuales. En este caso se puede afirmar que la evaluación se limita al resultado alcanzado por el trabajador en la ejecución de una tarea. Se trataría de una evaluación retrospectiva basada en consecuciones pasadas.

Por lo que se refiere a la evaluación de la actuación o desempeño (performance appraisal), cabe señalar que ésta se centra en la manera en que cada empleado realiza su trabajo. De este modo la evaluación tiene un carácter más subjetivo que la evaluación por resultados dado que es el evaluador quién determina el grado de ejecución de las tareas, o en qué medida el trabajador desarrolla los comportamientos deseados por la organización.

Otro factor que influye en el proceso de EDD es el conocimiento y/o acuerdo respecto a las actividades que hay que hacer, es decir, la claridad con respecto a los objetivos a conseguir. Las tareas con objetivos específicos y 
claros en los que los miembros de la organización y los responsables de los mismos se han puesto de acuerdo en cuanto a su ejecución y forma de medición son la base de un sistema de evaluación sencillo y eficaz. Igualmente el carácter de la evaluación cambiará en aquellas tareas que por su diseño o ejecución son fácilmente predecibles. Finalmente, la evaluación del potencial se entiende como un proceso que permite identificar posibilidades de desarrollo profesional en las personas de una organización (García-Tenorio, 2005:196). El fin último de esta evaluación es la de orientar al empleado hacia su perfeccionamiento, el diseño de planes de carrera o para la planificación y optimización de los recursos humanos. En este caso la organización trata de identificar las competencias que pueden llegar a tener los empleados en el futuro, para ocupar puestos de mayor responsabilidad en la escala.

La gestión por competencias es una faceta muy nueva y relevante dentro la gestión de recursos humanos que condiciona la forma en que se debe medir el rendimiento. La evaluación de las competencias profesionales implica atender a comportamientos observables, consecuencia de un conjunto de motivaciones, actitudes, valores, conocimientos, aptitudes, habilidades y destrezas (Vivas y Fernández, 2011: 44). Para Valle-Cabrera (2003), la evaluación por competencias sería necesaria, por ejemplo, para determinados puestos donde los resultados esperados sean difíciles de definir en términos precisos. Estas competencias serían una combinación de conocimientos y habilidades requeridas y resultados esperados en el desempeño de las tareas asignadas.

Los sistemas de gestión de rendimiento ofrecen, por tanto, información "feedback" tanto a los empleados como a los directivos. En el caso de los trabajadores, ello supone un mejor conocimiento de sus tareas y saber qué se espera de ellos; en el caso de los directivos, el sistema les ayuda a motivar y a mejorar la comunicación entre ellos y los trabajadores a su cargo. Igualmente, los sistemas de gestión del rendimiento facilitan el cambio organizativo, mejoran el diseño de los objetivos para los empleados y sus administradores así como reducen los comportamientos no deseados por parte de los empleados.

En la tabla 1 se indican los beneficios de un sistema de gestión del rendimiento.

\section{TABLA 1}

BENEFICIOS DE UN SISTEMA DE GESTIÓN DEL RENDIMIENTO

\section{Para los empleados}

- Mejora de su autoestima

- Mejor comprensión de los requerimientos y resultados esperados de sus puestos

- Mejor identificación de la forma de maximizar sus fortalezas y minimizar sus debilidades

\section{Para los directivos}

- Desarrollo de la mano de obra con una fuerte motivación hacia el rendimiento

- Mejor conocimiento de sus subordinados

- Logro de empleados más competentes

- Posibilidad de diferenciar más eficazmente los trabajadores con buenos y malos rendimientos

- Los directivos disfrutan de una comunicación más clara con sus trabajadores

\section{Para las organizaciones}

- Diseño más apropiado de sus acciones administrativas

- Establecimiento de objetivos claros para empleados y directivos

- Reducción de los comportamientos incorrectos de los empleados

- Disposición de mejor protección frente a demandas judiciales

- Mejora en la gestión del cambio organizativo

- Obtención de un mayor compromiso por parte de los empleados

- Consecución de una mayor participación de los empleados

FUENTE: elaboración propia a partir de Aguinis, H.; Joo, H. y Gottfredson, R. K. (2011): "Why we hate performance Management and why we should love it", Business Horizons, 54: 505. 


\section{Limitaciones inherentes al proceso de evaluación del desempeño}

La tarea de evaluar el desempeño no es fácil. Se evalúan personas y los evaluadores son también personas que normalmente forman parte de la organización. Estas cuestiones, como afirma De la Calle y Ortiz (2004), introducen cierta subjetividad en la evaluación y las reacciones, a veces conflictivas, entre individuos que trabajan juntos.

Argumentaciones similares podemos encontrar en Bowman (1999), Leal (2004) y Gómez-Mejía (2008). Estos "errores" del sistema atentan contra la objetividad del proceso. Por ello, el conocimiento y toma de conciencia de los mismos, antes de realizar la valoración, proporcionará una mayor fiabilidad y validez al sistema. Evaluar el rendimiento es complejo y depende de muchos factores, algunos de los cuáles van más allá del control del trabajador. Sin embargo, los observadores tienden a centrarse en él sobre todo cuando hay mal rendimiento. En este sentido, Bowman (1999) va más allá al argumentar que cuando ocurren los errores en la evaluación no sólo está en cuestión la validez del juicio del evaluador, sino que el resultado de la evaluación puede impedir al trabajador evaluado tener una valoración justa de su rendimiento.

Siguiendo a De la Calle y Ortiz (2004: 234), podemos decir que los principales problemas que podemos encontrar vienen de las relaciones entre las personas de la organización, fundamentalmente de la relación superiorsubordinado, de errores en el desarrollo de la evaluación y errores en la selección o aplicación de los métodos de evaluación.

Aunque los miembros de una organización trabajen para lograr los objetivos de la misma, no podemos olvidar que cada individuo tiene sus propios intereses y aspiraciones. Igualmente puede ser el propio evaluador quien asuma la responsabilidad de la evaluación sin haber recibido la formación necesaria (Longo, 2005). En estas situaciones aparece el efecto sesgo actor-observador, motivado por la tendencia del evaluador a buscar las razones del bajo rendimiento en el propio trabajador, mientras que éste justifica su rendimiento en factores (Carson, Cardy y Dobbins, 1991). Los errores en el desarrollo de la evaluación suelen estar fundamentalmente asociados a la condición humana de los evaluadores. Entre los más relevantes, podemos citar el efecto halo, el error de lenidad, error de severidad o el error de tendencia central (Bohlander, 2003; Sastre, 2003; Dolan, 2007; Dessler, 2009). Por último, los errores en la selección o aplicación de los métodos de evaluación provocan que aquello que se mide aparezca frecuentemente desconectado de las prioridades estratégicas de la organización (Longo, 2005).

Las organizaciones deben reducir al mínimo estos errores para conseguir una evaluación del rendimiento lo más precisa posible. Una manera de contrarrestar estos errores podemos encontrarla en London et al. (2004). Para estos autores, una forma de mejorar la precisión del evaluador por parte de las organizaciones se encuentra en la diferenciación de las evaluaciones, el uso de instrumentos de evaluación apropiados y en la formación del evaluador.

En este sentido, Curtis et al. (2005) proponen que la organización, para evitar los errores comentados determine correctamente la finalidad de la evaluación (fines meramente administrativos: retribución, formación, medidas disciplinarias, etc., o de mejora profesional del empleado a partir del feedback de la evaluación) y el instrumento de medida oportuno. Igualmente se debe considerar que la responsabilidad del evaluador, en todo proceso de evaluación del desempeño, es relevante para disminuir los errores en las calificaciones de rendimiento.

\section{LA GESTION DE RECURSOS HUMANOS EN LA ADMINISTRACIÓN PÚBLICA. LA NUEVA GESTIÓN PÚBLICA}

A lo largo del tiempo, se han producido cambios en las actividades del Estado, pasando de ser meramente garantistas a prestacionales. La gestión del sector público es claramente compleja debido a la diversidad de actividades y a su tamaño (Albi et al., 1997). Ciertamente, la gestión pública es, en el fondo, gestión dentro del sector público con sus características diferenciadoras. Las profundas transformaciones en el ámbito de las Administraciones públicas, producidas desde la década de los ochenta, determinan la necesidad de articular nuevos procedimientos en las relaciones de dichas Administraciones con los usuarios de los servicios públicos y la introducción de la idea de que la permanencia de las instituciones públicas debe venir condicionada por la obtención de ciertos resultados (Yerga, 2003).

Los procesos de reforma en las administraciones públicas, llevados cabo en los países de nuestro entorno, han sido estudiados por diversos autores (Pollitt, 2000; Pollitt, 2002; Box, 2001; Page, 2005; Minvielle, 2006; O'Flynn, 2007; Percebois, 2007; Corte-Real, 2008), existiendo consenso en cuanto a los elementos generales que conforman este "nuevo modelo" de gestión (Tabla 2). 
TABLA 2

\section{PRINCIPALES ELEMENTOS FORMALES QUE CONFORMAN EL NUEVO MODELO DE GESTIÓN}

- Un cambio en el enfoque de los sistemas de gestión que deberán estar más centrados en los productos y en los resultados que en los factores y en los procedimientos.

- Un cambio que implemente sistemas de valoración e indicadores de rendimiento estandarizados.

- Preferencia por organizaciones más especializadas, "delgadas" y horizontales frente a las grandes y multifuncionales burocracias jerárquicas.

- Uso de mecanismos de mercado más amplios (privatizaciones, subcontrataciones, desarrollo de mercados internos, etc.) para la prestación de los servicios públicos.

- La ampliación y difuminación de la frontera entre los sectores público y privado, caracterizado por el aumento de acuerdos de colaboración público/privados y la proliferación de organizaciones de carácter "híbrido".

- Un desplazamiento en cuanto a la prioridad de valores como el universalismo, la igualdad o la seguridad hacia la eficiencia y el individualismo.

FUENTE: elaboración propia a partir de Pollitt (2002): 474

El cambio en la contextualización de la sociedad, caracterizada por la globalización, la incertidumbre, la complejidad, la dinamicidad, la máxima incertidumbre, la pluralidad, la sociedad de la información y del conocimiento, la retroalimentación y la innovación, exige un cambio en los paradigmas sobre los que se ha asentado la Gestión Pública. Es así como surge la Nueva Gestión Pública, cuyas ideas principales giran en torno a la pretensión de aproximar las formas de producción públicas a las propias del sector privado, introduciendo técnicas gerenciales privadas reconociendo las peculiaridades de lo público (González, 2008: 9).

El término Nueva Gestión Pública (en adelante NGP), siguiendo a Olías de Lima (2001), responde por tanto, al intento de dar cuenta, reflexionar y discutir sobre cómo alcanzar fines públicos con la mayor eficiencia, eficacia y satisfacción del ciudadano.

En el concepto de NGP destacan tres ideas principales (Ballart, 2001): en primer lugar cabe señalar que, en ocasiones, los objetivos sociales de las organizaciones públicas se alcanzan mejor con financiación y regulación pública, pero cuya gestión se realiza a través de organizaciones privadas (o públicas que actúen con criterios empresariales y de competencia). Esta reforma pasa por la financiación flexible de las actuaciones públicas unido a sistemas de participación de los ciudadanos (gobernanza local) y de responsabilidad de los agentes intervinientes sobre los resultados obtenidos (Page, 2005); en segundo lugar, las actuaciones públicas son el resultado de decisiones que se adoptan en el seno de organizaciones públicas, a partir de ciertos procedimientos y siguiendo unos controles determinados. Las decisiones sobre los objetivos se toman por los órganos políticos y la Administración ejecuta sus funciones con la responsabilidad de tener a los ciudadanos como clientes de las intervenciones realizadas, ofreciéndoles el mejor servicio; finalmente, se debe tener en cuenta que el nuevo gestor, o directivo público, ha de ejecutar en este contexto, directrices políticas con criterios de servicio coste-efectivo.

La gestión pública tiende así a la obtención de la máxima productividad de los recursos escasos empleados con un modelo de gestión empresarial en un marco público, que permita responsabilizar a los gestores de los resultados y motivarles adecuadamente (Ortún, 1992).

Estas tendencias, en su conjunto, dan forma a una visión genérica del futuro del sector público. Así, para Percebois (2007:157), desde la década de los 70, la tendencia se ha invertido, y una gran mayoría de los países de la OCDE han decidido promover un sector público más pequeño pero más eficiente, a partir de lo que se conocen como las teorías de la Nueva Gestión Pública.

Actualmente, las reformas, tienen como fin último la mejora del rendimiento y de la eficiencia de los agentes e instituciones públicas, también pretenden realizar reducciones en los presupuestos, con el consiguiente ahorro en costes y la mejora de los procesos de prestación de servicios públicos, a partir del incremento en la capacidad, flexibilidad y elasticidad de la Administración Pública en su conjunto (Pollitt, 2000). Se trata de conjugar el "reino de los beneficios" con el "reino de los costes" (Olías de Lima, 2001), todo ello rodeado de sistemas de control que otorguen una plena transparencia de los procesos, planes y resultados, para que, perfeccionen el sistema de elección y favorezcan la participación ciudadana (García, 2007:37-64). 
Siguiendo a Albi et al. (1997) y a López (2003), debemos considerar que una Administración Pública que pretenda adoptar la nueva perspectiva de gestión eficiente de los servicios públicos deberá concentrar su atención en el desarrollo de un cambio cultural sobre la base de estos nuevos principios.

La gestión eficiente de los servicios públicos implica prestar atención a los circuitos de adopción de decisiones migrando de un enfoque tradicional de planificación y decisión a otro basado en los principios de la dirección por objetivos (DPO). Además, se trata de gestionar recursos humanos a través de su selección, pero también con su formación, capacitación, diseño de su carrera profesional y con sistemas de evaluación del rendimiento (Vivas y Fernández, 2011: 44). Desde la óptica de la gestión pública, las carreras profesionales no deberían estar tan cerradas como para imposibilitar la introducción de factores clave en la motivación, como son los elementos retributivos o las políticas de promoción, dentro de las restricciones financieras imperantes y del grado de estabilidad que se desee dar al empleo público. Es necesario flexibilizar las organizaciones, tanto desde un punto de vista puramente "organizativo" como jurídico, pues, contrariamente a lo que ha sido la tónica dominante en el pasado, cada vez son más numerosos los cambios en las configuraciones de las organizaciones públicas como un elemento más de persecución de la eficiencia. Por último, y a diferencia de la óptica administrativa, lo importante para la gestión es evaluar resultados más que realizar un seguimiento de los procedimientos utilizados. Esto implica un importante esfuerzo de medición de la actividad pública (Heinrich, 2002).

En definitiva, la gestión exige "ajustar" el diseño institucional, los estilos de dirección y los organigramas organizativos al cambio en los contextos en los que se desarrolla la actividad pública.

\section{La gestión de recursos humanos en la Administración Pública}

La gestión de los recursos humanos (en adelante, GRH) es un área clave para el buen funcionamiento de la organización que pretenda la excelencia en la oferta de sus productos y servicios (Longo, 2005; Villoria y del Pino, 2009; Barba y Serrano, 2012). La GRH se ocupa de uno de los activos más importantes de la organización: las personas. Dicha gestión puede definirse como una inversión en las personas para obtener su mayor utilidad, lo que en términos organizativos se traduce en la consecución de los resultados planificados por la organización (García, 2007; Serrano, 2010; Vivas, 2011). Este proceso, como se ha visto anteriormente, está repleto de actividades encaminadas a obtener y coordinar los recursos humanos de la organización. La forma de organizar y ordenar los recursos humanos es el primer elemento y escalón del sistema de empleo público. Definir el tipo de organización que queremos, delimitando su campo de actuación, conocer los principios organizativos que la inspiran, etc., va a influir en la regulación jurídica de la función pública (Yerga, 2003).

El tratamiento de los recursos humanos en el ámbito de la Administración Pública está adquiriendo cada vez más importancia. Se ha evolucionado de forma paulatina de una "administración de personal" (basada en contratos laborales, reclutamiento, gestión de nóminas y seguros sociales, tramitación de vacaciones y permisos, adiestramiento en actividades estandarizadas o habilidades del puesto) a una "gestión integral de recursos humanos", que tiene como referente el desarrollo de las personas en la organización, la adecuación de los regímenes de personal a las características de las tareas que tienen que desarrollar las diferentes organizaciones públicas, y el aumento constante de la profesionalización a través principalmente de mecanismos como: la provisión de puestos, el sistema de carrera, la formación y las retribuciones (OCDE, 1995).

En consecuencia, parece necesario, poner en marcha una nueva gestión de recursos humanos de carácter más descentralizado, incorporando técnicas de gestión privadas como las del tipo de "dirección por objetivos", sistemas de dirección estratégica, programas de gestión de la calidad o de la excelencia organizativa (Modelo Europeo de Gestión de Calidad, EFQM) o la implementación de técnicas directivas de control de gestión o de evaluación del rendimiento (Modelo Europeo de Excelencia para Organismos Públicos, MEEOP) (García, 2007).

\section{LA EVALUACIÓN DEL DESEMPEÑO EN LAS ADMINISTRACIONES PÚBLICAS}

Los estudios realizados en lo últimos años sobre la reforma de la función pública demuestran la preocupación existente sobre la EDD (Muñoz, 1999; Ministerio de Administración Pública, 2000; Longo, 2005; Walker y Boyne, 2009; Torres; Pina y Martí, 2010; Serrano y Barba, 2010).

Actualmente, la presión hacia el cambio o la "adaptación del paradigma burocrático" es imparable (Villoria, 2009: 572). A la administración ya no se le pide solo que actúe conforme a las leyes; además, se le demanda eficacia, eficiencia, equidad en el trato, calidad en el servicio, etc. Por todo ello la Administración española debe incorporar a su gestión el control del rendimiento y la evaluación como parte de sus actuaciones cotidianas.

El estudio de la medida del rendimiento de los empleados en las administraciones públicas es un tema de investigación novedoso en el ámbito de los recursos humanos (Cederblom y Pemerl, 2002; McAdam et al., 2005; 
de Groot y van Hulst; Heinrich, 2005; Bilgin, 2007; Sole y Schiuma, 2010; Brown, 2010; Torres et al., 2010; Salgado y Cabal, 2011; Barba y Serrano, 2012).

Los requisitos a los que obliga la gestión del rendimiento basada en resultados (Heinrich, 2002), está incrementando la necesidad de evaluar las actividades realizadas por los empleados públicos en todos los niveles gubernamentales, con instrumentos de medida que realmente sean útiles para el directivo público y efectivos, para mejorar el rendimiento de las actuaciones de los gobiernos. El "Libro blanco para la mejora de los servicios públicos" (2000:119) reconoce que el desarrollo profesional de los empleados públicos ha de estar basado necesariamente sobre dos claves: el conocimiento experto y el desempeño.

La gestión del rendimiento no es un fin en sí mismo sino un instrumento que, conectado con otras herramientas de gestión de personas, facilita la consecución de los objetivos organizativos. En este sentido, como recuerdan Villoria y del Pino (2009), los directivos de la organización son los responsables de la consecución de los objetivos organizativos y los gestores de niveles inferiores tienen encomendada la consecución de los objetivos respectivos de sus unidades. Por ello, la gestión del rendimiento supone el enlace entre el "qué" (objetivo, resultado y estándar de rendimiento) y el "cómo" (proceso, competencia y comportamiento) del rendimiento del individuo (Bilgin, 2007). Es decir, la EDD debe considerar también las conductas que tienen que emplear las personas para cumplir con los objetivos, así como el grado de adquisición de competencias óptimas para mejorar y optimizar los procesos de gestión en la organización (Vivas, 2011).

La utilidad de la evaluación del rendimiento se puede entender en un doble sentido: interno y externo (Torres, 2010). Desde el punto de vista interno, el uso de sistemas de medida del rendimiento crea incentivos que ayudan a alinear los objetivos individuales con los de la administración, marcando el progreso de consecución de dichos objetivos y sentando las bases para una mejor, y más efectiva, toma de decisiones. Desde el punto de vista externo, la utilización de herramientas de medida del rendimiento se corresponde con la idea de transparencia y de responsabilidad que las administraciones públicas deben dar hacia los ciudadanos (Sole y Schiuma, 2010). No debemos olvidar que en las actuaciones de la Administración tan importante es el cumplimiento de los objetivos, como la forma en la que se cumplen dichos objetivos (Villoria y del Pino, 2009).

Por tanto, y siguiendo a Yerga (2003), podemos afirmar que la finalidad del sistema de evaluación del desempeño en la Administración Pública debe permitir:

Conocer el rendimiento del empleado público, tanto individual como en conjunto con su unidad de trabajo, en relación con los objetivos de la organización en que prestan sus servicios.

Determinar sus requerimientos, insuficiencias, posibilidades de desarrollo y necesidades de formación, proporcionando información relevante en orden al diseño de carrera profesional o régimen disciplinario, entre otros.

Proporcionar información a los empleados públicos a fin de que conozcan su desempeño y sugerirles los cambios que necesitan realizar en sus comportamientos, actitudes o capacidades en relación con los objetivos de la organización.

Integrar los intereses individuales con los del organismo público, mejorando su sentido de afiliación y pertenencia.

\section{LA EVALUACIÓN DEL DESEMPEÑO EN EL ESTATUTO BÁSICO DEL EMPLEADO PÚBLICO}

El Estatuto Básico del Empleado Público (en adelante, EBEP) fue aprobado por Ley 7/2007, de 12 de abril. En la función pública española se han producido reformas parciales y reformas integrales. Echando una mirada al pasado, esta reforma de la Ley 7/2007 sería la cuarta reforma integral de la Función pública en España (Pinto, 2009:107-108)'. Sin duda, se puede afirmar que constituye un importante paso hacia adelante. Pese a la vocación integral que tiene el Estatuto, cabe afirmar que mientras no se produzca el desarrollo completo de éste, ya sea a través de la legislación estatal o autonómica, no se puede afirmar que el marco regulador del empleo público está completo. Como señalan Sánchez y Molina (2012), el desarrollo del EBEP no se ha producido ni a nivel estatal ni en la mayoría de las Comunidades Autónomas².

1 Con anterioridad a esta Ley, las reformas fueron las siguientes: el Estatuto de Maura de 1918, la reforma de Bravo Murillo de 1852, y, finalmente, la reforma de la Ley de los Funcionarios Civiles del Estado de 1964.

2 Las Comunidades que han aprobado legislación de desarrollo del EBEP son una minoría. Concretamente, se pueden citar la Comunidad Valenciana y la Comunidad de Castilla-La Mancha. Ello ha supuesto la aprobación de la Ley 10/2010, de 9 de julio de la Generalitat, de Ordenación y Gestión de la Función Pública Valenciana; Ley 4/2011, de 10 de marzo, de Empleo Público de Castilla-La Mancha, respectivamente. Estas leyes de desarrollo no han tratado todos los aspectos que establece el EBEP. 
El objetivo del EBEP es modernizar el régimen jurídico de la Función Pública española, incorporando una serie de cambios sustanciales respecto de la normativa anterior. Entre dichos cambios, cabe destacar la introducción del concepto evaluación del desempeño del empleado público. La evaluación del desempeño supone un eje fundamental y transversal del nuevo modelo de empleo público, y de una mayor profesionalización de los empleados públicos.

La importancia atribuida a la evaluación del desempeño en el EBEP se produce ya en el Informe de la Comisión para el estudio y preparación del Estatuto Básico del Empleado Público. Concretamente, refiriéndose a la evaluación del desempeño, la Comisión considera que ésta debe ser un elemento central de cualquier reforma modernizadora de nuestras Administraciones Públicas³. En la misma línea, la Exposición de Motivos del EBEP, considera que la evaluación del desempeño de los empleados públicos es un elemento fundamental y a lo largo del articulado del Estatuto, se hace referencia a la evaluación del rendimiento en diversos artículos.

Para nuestro análisis, tomamos como punto de partida el concepto de evaluación del rendimiento que establece el EBEP. El citado Estatuto define la evaluación del desempeño como el procedimiento mediante el cual se mide y valora la conducta profesional y el rendimiento o logro de resultados. De acuerdo con esta definición, en la evaluación del desempeño es fundamental centrarse en la conducta profesional y la consecución de resultados o rendimiento.

González y Herrero (2011:55-56), refiriéndose al EBEP, consideran que la evaluación del desempeño es una potente herramienta de gestión de recursos humanos, que tiene como base los siguientes principios informadores:

- La evaluación del desempeño es de obligatoria implantación. El EBEP señala que "las Administraciones Públicas establecerán sistemas que permitan la evaluación del desempeño de sus empleados”.

- Se trata de un procedimiento "mediante el cual se mide y valora la conducta profesional y el rendimiento o logro de resultados".

- Se establecen los requisitos generales que deben reunir estos sistemas de evaluación del desempeño, determinando que "se adecuarán en todo caso a los criterios de transparencia, objetividad, imparcialidad y no discriminación y se aplicarán sin menoscabo de los derechos de los empleados públicos”.

- Se determina la conexión con la relación jurídica de empleo público. El EBEP establece que “las Administraciones Públicas determinarán los efectos de la evaluación del desempeño en la carrera profesional horizontal, la formación, la provisión de puestos de trabajo y en la percepción de retribuciones complementarias". También dispone que la continuidad de un puesto de trabajo obtenido por concurso quedará vinculada a la misma.

Por todo lo que implica la evaluación del rendimiento, nos parece acertada la opinión de Fuentetaja al considerar que la evaluación del rendimiento es, probablemente, el reto más arduo de implantar que plantea el EBEP (2007: 495).

\section{Efectos de la gestión de la evaluación del desempeño para las administraciones públicas}

De acuerdo con el EBEP, las administraciones públicas determinarán los efectos de la evaluación en base a los siguientes aspectos: la carrera profesional horizontal, la formación, la provisión de puestos y la continuidad en un puesto de trabajo obtenido por concurso, y la percepción de las retribuciones complementarias previstas en el art. 24 del EBEP.

Con respecto a la carrera profesional, y relacionado con el tema de la evaluación del rendimiento, interesa destacar que, de acuerdo con el artículo 17 del EBEP, las Leyes de Función Pública que se dicten en desarrollo del presente Estatuto podrán regular la carrera horizontal de los funcionarios de carrera. De este modo, se podrá aplicar la siguiente regla establecida en el apartado b) del citado artículo: "Se deberá valorar la trayectoria y actuación profesional, la calidad de los trabajos realizados, los conocimientos adquiridos y el resultado de la evaluación del desempeño. Podrán incluirse asimismo otros méritos y aptitudes por razón de la especificidad de la función desarrollada y la experiencia adquirida".

En consecuencia, la carrera horizontal se encuentra vinculada a la evaluación efectuada al empleado y, como señala Villoria y del Pino (2009), de ello se desprende la enorme importancia que la evaluación tendrá para la consolidación de las categorías personales.

\footnotetext{
3 Informe de la Comisión para el estudio y preparación del Estatuto Básico del Empleado Público, p. 107.
} 
En relación a la formación, el art. 20.3 del EBEP se ocupa de los efectos de la evaluación en la formación de los empleados. El citado Estatuto hace referencia a la formación en tres artículos. En primer lugar, el Estatuto establece como derecho individual de los empleados públicos el derecho "a la formación continua y a la actualización" (art. 14, g). También cuando se refiere a las medidas objeto de negociación colectiva estableciendo como una de ellas “los criterios generales de los planes y fondos para la formación y la promoción interna” [(art. 37, f)]. Por último, el Estatuto al tratar los principios de conducta dispone que "los empleados públicos mantendrán actualizada su formación profesional” (art. 54.8). Con ello se pretende, básicamente, la identificación de cuáles son los déficits de conocimientos, habilidades o aptitudes detectadas, con el propósito de corregirlos mediante los pertinentes programas formativos (Pinto, 2008).

La regulación de la provisión de puestos de trabajo se realiza en los artículos 78 y 79 del EBEP. De acuerdo con el Estatuto, el procedimiento normal de provisión de puestos de trabajo “consistirá en la valoración de méritos y capacidades y, en su caso, aptitudes de los candidatos por órganos colegiados de carácter técnico” (art. 79).

Como señala González y Herrero (2011), se ha criticado que en este artículo no se haga mención alguna a la evaluación del desempeño. Esta omisión debería ser objeto de enmienda por la legislación.

Finalmente, el Estatuto dispone que las Administraciones Públicas determinarán los efectos de la evaluación del desempeño en la percepción de las retribuciones complementarias previstas en el artículo 24 del mismo Estatuto (art. 20.3). Este sistema de retribución abierto es coherente con el sistema de carrera que también busca el Estatuto (Pinto, 2008).

El citado art. 24 señala que la cuantía y estructura de las retribuciones complementarias de los funcionarios se establecerán por las correspondientes leyes de cada Administración Pública, atendiendo, entre otros, a los siguientes factores:

a) La progresión alcanzada por el funcionario dentro del sistema de carrera administrativa.

b) La especial dificultad técnica, responsabilidad, dedicación o incompatibilidad exigible para el desempeño de determinados puestos de trabajo o las condiciones en que se desarrolla el trabajo.

c) El grado de interés, iniciativa o esfuerzo con que el funcionario desempeña su trabajo y el rendimiento o resultados obtenidos.

d) Los servicios extraordinarios prestados fuera de la jornada normal de trabajo.

Dado que en la regulación normativa del EBEP no se hace una referencia expresa a la evaluación de desempeño, Linde (2008) considera que los apartados b) y c) del artículo 24 son versiones matizadas de la evaluación del desempeño, mientras que los apartados a) y d) supondrían factores ajenos a éste.

\section{La evaluación del rendimiento en el EBEP: ¿gestión por objetivos o adquisición de competencias?}

De acuerdo con el artículo 20 del EBEP, la “evaluación del rendimiento mide y valora la conducta profesional y el rendimiento o logro de resultados". En opinión de Villoria y del Pino (2009), en principio el logro de resultados implica evaluar por objetivos, es decir, la denominada gestión por objetivos. No obstante, con respecto a la conducta profesional, no queda tan claro como se evalúa ésta. La conducta profesional podría quedar vinculada al sistema de factores o a la adquisición de competencias. Para dar respuesta a esta cuestión, será preciso esperar al desarrollo completo de la normativa correspondiente al EBEP.

La cuestión antes comentada tiene importancia porque evaluación de resultados y evaluación del desempeño son cuestiones distintas cómo se ha comentado anteriormente en este trabajo. Siguiendo a Cortés (2009), cabe afirmar que la evaluación de resultados nos da información acerca de si el empleado tiene o no éxito en su puesto, incluso puede facilitar información, con la utilización de indicadores, acerca de si el rendimiento de un empleado es más alto que el de otro. Sin embargo, no nos facilita información sobre por qué el rendimiento es mejor o cómo podemos mejorar la productividad de aquéllos que no tienen rendimientos tan altos. En cambio, el concepto de desempeño hace referencia a "la forma de hacer", a todo aquello que contribuye o que hace que un empleado tenga éxito.

Podemos considerar que la evaluación por objetivos pretende medir la contribución y grado de implicación del personal público al cumplimiento de los objetivos anuales establecidos para la unidad a la que pertenecen. En cambio, la evaluación por competencias de los empleados públicos se debe realizar en función a los perfiles de exigencias establecidos para sus puestos de trabajo. Esta evaluación se puede realizar a través de la valoración de la conducta profesional de los empleados, observada a través de sus comportamientos en el trabajo. 
Villoria y del Pino (2009) dudan acerca de si en la legislación de desarrollo se incorporarán dimensiones relacionadas con el desempeño o si, por el contrario, se centrará sólo en las tareas y resultados. Este autor, siguiendo a Sacket y Devore (2001), realiza la siguiente agrupación de las dimensiones: desempeño de tareas o comportamientos necesarios para realizar la tarea, desempeño contextual o conductas productivas y conductas contraproductivas.

\section{Procedimiento y órgano de evaluación}

Con respecto al procedimiento de evaluación al que se refiere el artículo 20.1 del EBEP, siguiendo a Linde (2008), se pueden establecer una serie de conclusiones: en primer lugar, dicha evaluación se llevará a cabo a través de procedimientos especiales. Estos procedimientos serán el resultado de la legislación estatal, de las Comunidades Autónomas y de los convenios colectivos; en segundo lugar, el EBEP no señala si el procedimiento de evaluación será único o a través de múltiples procedimientos, aunque sí parece cierto el carácter especial que se predicará de dichos procedimientos; y, en tercer lugar, cabe significar que el procedimiento de evaluación requiere, con carácter previo, concretar unidades de medida y de valoración. El EBEP no establece ninguna referencia a cuáles serán dichas unidades. Por tanto, será la legislación de desarrollo la encargada de establecerlas. Tanto las unidades de valoración como las de evaluación deberán ser conocidas por los empleados públicos, con anterioridad al período de evaluación.

En lo referido al órgano de evaluación, el EBEP no contiene ninguna mención en su articulado. Las posibilidades para el establecimiento de la persona u órgano de evaluador son múltiples. Linde (2008) se muestra claramente partidario de un órgano colegiado de evaluación. En su opinión, dicho órgano no debería estar sometido al principio de jerarquía funcional en relación con los órganos superiores y directivos de los distintos departamentos ministeriales. Coincidimos con este autor en la importancia de que el órgano sea colegiado, pero estimamos que si debería participar, siquiera mínimamente, el superior jerárquico del evaluado. La participación del superior jerárquico puede ser interesante para fines aclaratorios, de precisión de determinadas cuestiones, de valoración, etc. Estos procedimientos de evaluación deberán contemplar la posibilidad de impugnación por parte del evaluado.

\section{EI EBEP tampoco hace referencia a la frecuencia temporal de la evaluación del rendimiento.}

Sin duda, la puesta en marcha de estos procedimientos de evaluación exigirá un gran esfuerzo de formación entre los gestores de recursos humanos en el seno de las Administraciones Públicas. La profesionalización de la gestión está muy presente en las intenciones del propio EBEP (Fuentetaja, 2007: 497). En nuestra opinión, los gestores de recursos humanos están llamados a ocupar un papel fundamental en la aplicación del EBEP y la normativa que lo desarrolle.

La puesta en práctica del sistema de evaluación del desempeño se debe considerar como un reto y una oportunidad para lograr que la Administración Pública sea más eficaz y eficiente. El EBEP no ha sido desarrollado desde el punto de vista estatal. Respecto al desarrollo del EBEP a nivel de Comunidades Autónomas, cabe afirmar que éste ha sido muy limitado. Se puede destacar la Ley 10/2010, de 9 de julio, de la Generalitat, de Ordenación y Gestión de la Función Pública Valenciana y la Ley 4/2011, de 10 de marzo, de Empleo Público de Castilla-La Mancha.

Con respecto a la Ley 10/2010, de 9 de julio, de la Generalitat, de Ordenación y Gestión de la Función Pública Valenciana, cabe recordar que el art. 121 de la citada Ley se ocupa de la evaluación del desempeño, pero remite al desarrollo reglamentario la determinación del sistema y el procedimiento para la evaluación y la composición y funcionamiento de la comisión técnica encargada de la misma, así como los efectos de su resultado sobre la carrera horizontal, la formación, la provisión de puestos de trabajo y, en su caso, la percepción del complemento de actividad profesional. También se establece que la continuidad del personal funcionario de carrera en los puestos de trabajo obtenidos por concurso, quedará vinculada al resultado de la evaluación, de acuerdo con el sistema y el procedimiento que se determine reglamentariamente, dándose audiencia a la persona interesada y a través de la correspondiente resolución motivada. Por tanto, quedan muchas cuestiones remitidas al desarrollo reglamentario, que aún no se ha producido.

Con respecto a la Ley 4/2011, de 10 de marzo, de Empleo Público de Castilla-La Mancha, cabe señalar que el art. 66 se ocupa de la evaluación del rendimiento, pero realiza una remisión al desarrollo reglamentario de dicha Ley. Actualmente, dicho desarrollo aún no se ha producido.

La actual situación de reformas en las que se encuentra inmerso nuestro país debe suponer una oportunidad para que se produzca el desarrollo del EBEP en materia de evaluación del rendimiento. Urge realizar el desarrollo completo del mismo, siendo especialmente interesante que se regule de forma más concreta el tema de la evaluación del rendimiento. 


\section{CONCLUSIONES}

En este trabajo se han analizado las ventajas que para el conjunto de las organizaciones tienen los sistemas de evaluación del desempeño, facilitando el cambio organizativo, mejorando el diseño de los objetivos para los empleados y sus administradores así como reduciendo los comportamientos no deseados por los empleados, entre otros. Cabe señalar que del estudio y conocimiento de las limitaciones de dicho proceso es posible configurar sistemas más efectivos y que generen menos incertidumbres entre los empleados y evaluadores.

La gestión de los recursos humanos se ha convertido en una de las principales preocupaciones en el ámbito empresarial. Por ello, consideramos que desde el campo de estudio de la Organización de Empresas se puede realizar un aporte fundamental al ámbito del sector público, dónde cada vez gana más importancia la gestión de los recursos humanos.

La rapidez de los cambios experimentados en el ambiente de las organizaciones, la globalización de los mercados y su mayor competitividad ponen de manifiesto la necesidad de una nueva cultura en la gestión de las organizaciones, obligando a las mismas a realizar ajustes internos y a adaptarse rápidamente a dichos cambios de una forma innovadora y/o creativa.

Todos estos nuevos requerimientos están fuertemente relacionados con el factor humano, situándolo como un factor determinante y, al mismo tiempo, como un recurso estratégico para la organización. Sin duda, la correcta gestión de los recursos humanos se ha convertido en una de las claves más importantes para lograr el éxito.

Partiendo de la complejidad que envuelve al Sector Público, la Administración Pública debe adoptar un planteamiento próximo al que se viene realizando de manera exitosa en las organizaciones privadas, con el fin de mejorar la gestión de recursos humanos.

El carácter integral que tiene en la actualidad la gestión de los recursos humanos obliga, en cierta manera, a que la evaluación del desempeño esté irremediablemente enlazada con otros aspectos esenciales en las políticas de personal de la Administración y, en concreto, con un nuevo modelo de empleo público que sea capaz de reclutar a profesionales con perfiles adecuados a la realidad actual, de motivar, de formar y actualizar a los servidores públicos para el cumplimiento eficiente y eficaz de sus funciones y responsabilidades. Ofreciéndoles, a la vez, suficientes oportunidades de promoción profesional, basada en una valoración objetiva de su desempeño y cualidades, y que facilite una gestión racional y objetiva, ágil y flexible del personal, atendiendo al continuo desarrollo de las tecnologías de la información.

La evaluación del rendimiento laboral es un aspecto relevante de la gestión de personal que las organizaciones del sector público deben incorporar a sus pautas de funcionamiento, realizando los oportunos cambios entre Administración y empleados.

La evaluación del desempeño es una obligación que establece el Estatuto Básico del Empleado Público para todas las Administraciones Públicas españolas. El objetivo no es otro que el de modernizar el régimen jurídico de la Función Pública española, incorporando una serie de cambios sustanciales respecto de la normativa anterior, con conceptos novedosos como son la figura del personal directivo profesional, la carrera profesional horizontal y su vinculación con la retribución de los empleados públicos.

Por ello es necesario que el concepto de evaluación del desempeño, establecido en el EBEP, sea desarrollado reglamentariamente tanto a nivel estatal como de las Comunidades Autónomas. Este desarrollo permitirá dotar a las Administraciones Públicas de modelos de evaluación, aclarará algunos interrogantes en torno a la evaluación del rendimiento de los empleados públicos, y deberá suponer que la gestión de los Recursos Humanos públicos se encamine al logro de la eficiencia.

En este sentido no podemos dejar de constatar las muchas diferencias existentes entre los ámbitos público y privado, sobre todo en los modelos de gestión de recursos humanos. Las Administraciones Públicas se encuentran limitadas por el importante grado de burocratización existente, que merma considerablemente su autonomía. A ello hay que añadir el rechazo al cambio entre los empleados públicos y la menor flexibilidad que tiene el sector público.

Bajo éste marco teórico, y en base al objetivo marcado con este trabajo, podemos anticipar posibles líneas de investigación futuras en el ámbito de las organizaciones públicas, ya sea a partir de la adaptación del sistema de evaluación del rendimiento individual de la empresa a las exigencias y limitaciones de las organizaciones públicas, condicionadas por el régimen jurídico y el sistema de selección de personal, o bien mediante la elaboración de un patrón para la adaptación del sistema de evaluación del rendimiento individual adoptado en el ámbito empresarial al marco de las organizaciones públicas. 
Igualmente sería posible el estudio de las ventajas y mejoras que la implantación del sistema de evaluación del rendimiento puede aportar a la Administración Pública, para que ésta logre desempeñar su misión de una manera más eficaz y eficiente, a la vez de servir como instrumento válido para mejorar la eficiencia y calidad de la prestación del servicio público, así como la motivación, productividad e imagen social del empleado público.

Por último, la utilización de los sistemas de evaluación del rendimiento puede ser el instrumento que facilite la implantación de los principios de la Nueva Gestión Pública en las Administraciones, tal y como está ocurriendo con los modelos de gestión de la calidad total basados en el EFQM (European Foundation for Quality Management).

\section{BIBLIOGRAFÍA}

AGUINIS, H. (2009), Performance management, Upper Saddle River, NJ, Pearson Prentice Hall.

Aguinis, H.; Joo, H. y GotTfredson, R. K. (2011), "Why We Hate Performance Management and Why We Should Love It", Business Horizons, 54: 503-507.

ALBI, E.; GONZÁLEZ, J. y LÓPEZ, G. (1997), Gestión pública. Fundamentos, técnicas y casos. Ariel Economía, Barcelona.

AlBIZU, E. y LERTXUNDI A. (2011),"La dirección estratégica de los recursos humanos", en: Dirección estratégica de los Recursos Humanos, Coord. Albizu Gallastegui, E. y Landeta Rodríguez, J., Ed. Pirámide, 2011: 282.

BADAWY, M. (2007), “Managing human resources”, Research Technology Management, july-august 2007: 56-74.

BALLART, X. (2001), Innovación en la gestión pública y en la empresa privada, Ediciones Díaz de Santos, Madrid.

BARBA, M. I. y SERRANO, J. (2012), "Efecto de las prácticas de recursos humanos en los ayuntamientos”, en: XXII Congreso nacional de ACEDE, septiembre de 2012, Cádiz.

BARNEY, J. (1991), “Firm Resources and Sustained Competitive Advantage”, Journal of Management, 17(1): 99-120.

BARNEY, J. y DELWYN, N. (2007), "Firm Resources and Sustained Competitive Advantage”, en Resource-Based Theory. Creating and Sustaining Competitive Advantage, Oxford University Press, New York: 49-75.

BARNeY, J. y WRIGHT, P. (1998), “On Becoming a Strategic Partner: the Role of Human Resources in Gaining Competitive Advantage", Human Resource Management, 37: 31-46.

BILGIN, K. (2007), "Performance Management for Public Personnel: Multi-analysis Approach toward Personnel”, Public Personnel Management, vol. 36 (2): 93-113.

Bohlander, G.; SHERMAN, A. y SNELL, S. (2003), Administración de recursos humanos, Thomson, Madrid.

BowmAN, J. (1999), "Performance Appraisal: Verisimilitude Trumps Veracity”, Public Personnel Management, vol. 28 (4): 557-576.

Box, R. et al. (2001), "New Public Management and Substantive Democracy”, Public Administration Review, vol. 61 (5): 608-618.

BRINKERHOFF, D. y KANTER, R. (1980), “Appraising the Performance of Performance Appraisal”, Sloan Management Review, vol. 21 (3): 3-16.

Brown, M.; HyATt, D. y BEnson, J. (2010), “Consequences of the Performance Appraisal Experience”, Personnel Review, vol. 39 (3): 375-396.

CAMIsón, C. (1997), La competitividad de la PYME industrial española: estrategia y competencias distintivas, Civitas, Madrid.

CARROLL, S. J. y SCHNEIR, C. E. (1982), Performance Appraisal and Review Systems: the Identification, Measurement, and Development of Performance in Organizations. Glenview, Illinois, Scott Foresman Company.

CARSON, K.; CARDY, R. y DOBBINS, G. (1991), "Performance Appraisal as Effective Management or Deadly Management Disease: two Initial Empirical Investigations”, Group and Organization Studies, 16: 143-159.

Cederblom, D. y PeMERL, D. (2002), “From Performance Appraisal to Performance Management: one Agency's Experience”, Public Personnel Management, vol. 31 (2): 131-139.

CLIFFORD, Jr. y CAVANAG, R. (1985), “The Winning Performance: how America's High-growth Midsize Companies Succeed”, en Estrategias de éxito para la pequeña y mediana empresa, Ediciones Folio.

CORTE-ReAL, I. (2008), “Public Management Reform in Portugal: Successes and Failures”, International Journal of Public Sector Management, vol. 21 (2): 205-229.

GESTIÓN Y ANÁLISIS DE POLÍTICAS PÚBLICAS, Nueva Época, no 10 julio-diciembre 2013 ISSN: 1989-8991 - DOI: 10.24965/gapp.v0i10.10115 
CORTÉS, J. (2009), “La evaluación del desempeño en el Estatuto Básico del Empleado Público”, en El consultor de los ayuntamientos y de los juzgados, n 1, quincena 15-29 enero, referencia 36/2009: 36. Tomo I.

CURTIS, A.; HARVEY, R. y RAVDEN, D. (2005), "Sources of Political Distortions in Performance Appraisals. Appraisal Purpose and Rater Accountability", Group and Organization Management, vol. 30 (1): $42-60$.

DELERY, J y DOTY, D (1996), “Modes of Theorizing in Strategic Human Resources Management: Test of Universalistic, Contingency, and Configurational Performance Predictions", Academy of Management Journal, 39 (4): $802-835$.

De la Calle, M. y Ortiz de URBina, M. (2004), Fundamentos de recursos humanos, Pearson-Prentice Hall, Madrid.

DESSLER, G. (2009), Administración de recursos humanos, Pearson-Prentice Hall, Madrid.

DOLAN, S.; SHULER, R. y VALLE, R. (2007), La gestión de los recursos humanos, McGraw-Hill, Madrid.

ESPAÑA. MINISTERIO DE AdMINISTRACIÓN PÚBLICA (1991), Estudio Delphi. La modernización de los procedimientos de actuación en la Administración Pública. IGSAP, Madrid.

ESPAÑA. Ministerio de AdMINISTRACIÓN PúBLICA (2000), Libro blanco para la mejora de los servicios públicos: una nueva Administración al servicio de los ciudadanos, Madrid.

FERRIS, G.; HoCHWARTER, W.; BUCKLEY, M.; HARRELL-COOK, G. y FrINK, D. (1999), “Human Resources Managements: Some New Directions", Journal of Management, vol. 25 (3): 385-415.

FuentetAjA, J. (2007), “El Estatuto Básico del Empleado Público”, Revista de Administración Pública, núm 174, septiembre-diciembre: 457-499.

GARcíA-TEnorio, J. y SABATER, R. (coord.) (2005), Fundamentos de dirección y gestión de recursos humanos, Thomson Paraninfo, Madrid.

GarcíA, I. (2007), "La Nueva Gestión Pública: evolución y tendencias”, Presupuesto y Gasto Público, 47/2007: $37-64$.

GIL, I.; RUIZ, L. y RUIZ, J. (1997), La nueva dirección de personas en la empresa. McGraw-Hill, Madrid.

Gómez-MEJíA, L. R.; BALKIN, D.B. y CARDY, R.L. (2008), Gestión de recursos humanos, Pearson-Prentice Hall, Madrid.

GonzÁLEZ, M. (2008), “Concepto y evolución de Gestión Pública” en: La acción y los retos del Sector Público. Una aproximación, Ed. Delta, Madrid.

GonzÁlez de Herrero, P. y PÉrez-BARojA, I. (2011), “Plan de experiencias piloto en Evaluación del Desempeño en la Administración General del Estado”, en: Boletín de Función Pública del INAP, num.6: 55-62.

GRANT, R. (1991), “The Resource-based Theory of Competitive Advantage: Implications for Strategy Formulation", California Management Review, 33 (3): 114-135.

HEINRICH, C. (2002), “Outcomes-based Performance Management in the Public Sector: Implications for Government Accountability and Effectiveness”, Public Administration Review, vol. 62 (6): 712-725.

HeISLER, W., Jones, W. y BeNHAM, P. (1988), Managing Human Resourced Issues, San Francisco, Jossey-Bass, .

HERRERA, J. (2001), Dirección de recursos humanos. Un enfoque de administración de empresas. ACDE Eds., Valencia.

HUCK, J. y MCEWEN, T. (1991), “Competencies Needed for Small Business Success: Perceptions of Jamaican Entrepreneurs", Journal of Small Business Management, vol. 29 (2): 90-93.

HUSELID, M. (1995), “The Impact of Human Resource Management Practices on Turnover, Productivity, and Corporate Financial Performance”, Academy of Management Journal, 38: 635-670.

KoONTZ, H. y WeIHRICH, H. (1998), Administración, McGraw-Hill, México.

LEAL, A. (coord.) (2004), EL factor humano en las relaciones laborales, Pirámide, Madrid.

LINDE, E. (2008), “La evaluación del desempeño de los empleados en el Estatuto Básico del empleado público”, en Linde, E. (Dir.) El Estatuto Básico del Empleado Público y su desarrollo por el Estado y las Comunidades Autónomas, Ed. Colex, Madrid: 179-201. 
LONDON, M.; MONE, E. y Scott, J. (2004), “Performance Management and Assessment: Methods for Improved Rater Accuracy and Employee Goal Setting”, Human Resource Management, vol. 43 (4): 319-336.

LONGO, F. (2005), “Evaluación y gestión del rendimiento laboral en las Administraciones Públicas”, Presupuesto y Gasto Público, 41/2005: 127-144.

LóPEZ, G. (dir.) (2003), Los nuevos instrumentos de la gestión pública. Servicio de estudios de la Caja de Ahorros y Pensiones de Barcelona "La Caixa”.

LUCK, S. (1996), "Success in Hong Kong: Factors Self-reported by Successful Small Business Owners", Journal of Small Business Management, vol. 34 (3): 68-75.

MARTIN, D. y BARTOL, K. (1998), “Performance Appraisal: Maintaining System Effectiveness”, Public Personnel Management, vol. 27(2): 223-230.

MCADAM, R.; HAZLETT, S. y CASEY, C. (2005), "Performance Management in the UK Public Sector", The International Journal of Public Sector Management, vol.18 (3): 256-273.

MinVIELLE, E. (2006), “New Public Management a la Francaise: the Case of Regional Hospital Agencies”, Public Administration Review, vol. 66 (5): 753-763.

MONFORT, V. (2000), "Competitividad y factores críticos de éxito en la hostelería de litoral: experiencia de los destinos turísticos Benidorm y Peñíscola”, FITUR, 2000, Madrid.

MuÑoz, A (1999),La gestión de la calidad total en la administración pública. Ediciones Díaz de Santos, Madrid.

OCDE (1995), Flexibilidad en la gestión de personal en la Administración Pública. Instituto Nacional de Administración Pública, MAP, Madrid.

OLíAS DE LIMA, B. (coord.) (2001), La nueva gestión pública, Prentice Hall, Madrid.

O'FlYNN, J. (2007), “From New Public Management to Public Value: paradigmatic change and managerial implications", The Australian Journal Of Public Administration, vol.66 (3): 353-366.

ORTún, V. (1992), “Gestión pública”, Centro de estudios sobre economía del sector público. Centro de publicaciones de la Fundación BBVA, Madrid.

PAGE, S. (2005), "What's new about the New Public Management? Administrative Change in the Human Services", Public Administration Review, vol. 65 (6): 713-727.

Percebois, L. (2007), “Benefits without Drawbacks? Adverse or Complex Effects of Public Management Reforms”, Presupuesto y Gasto Público, 48/2007: 145-161.

PETERAF, M. y BARNEY, J. (2003), “Unraveling the Resource-based Triangle”, Managerial and DecisionEconomics, 24: 309-323.

PINTO, M. (2008), “Gestión y Recursos Humanos” en González, C. (Coord.) La acción y los retos del Sector Público. Una aproximación. Delta Publicaciones: 107-110.

POLLITT, C. (2000), "Is the Emperor in his Underwear? An Analysis of the Impacts of Public Management Reform”, Public Management, vol. 2 (2): 181-189.

POLlitT, C. (2002), "Clarifying Convergence. Striking Similarities and Durable Differences in Public Management Reform”, Public Management Review, vol. 4 (1): 471-492.

PUCHOL, L. (1997), Dirección y gestión de recursos humanos, Díaz de Santos, Madrid.

PfEFfER, J. (1994), “Competitive Advantage through People”, California Management Review, 36: 9-29.

RoBerTS, G. (2002), “Employee Performance Appraisal System Participation: a Technique that Works”, Public Personnel Management, vol. 31 (3): 333-342.

SACKETT, P. y DeVORE, C. (2001), “Conterproductive Behaviors at Work”, en ANDERSON, N.; ONES, D.; SINAGIL, H. y VISWESVARAN, C. (eds.), Handbook of Industrial and Organizational Psycology, n 1, SAGE, Londres: 145-164.

SALGAdO, J. y CABAL, A. (2011), "Performance Appraisal in the Public Administration of the Principality of Asturias: Analysis of Psychometric Properties”, Revista de Psicología del Trabajo y de las Organizaciones, vol.27 (2): $75-91$.

SÁNCHEZ, M. y MolinA, D. (2012), “El desarrollo legislativo del EBEP en las Comunidades Autónomas”, X Seminario de Aspectos Jurídicos de la Gestión Universitaria, Universidad de El País Vasco.

GESTIÓN Y ANÁLISIS DE POLÍTICAS PÚBLICAS, Nueva Época, n 10 julio-diciembre 2013 ISSN: 1989-8991 - DOI: 10.24965/gapp.v0i10.10115 
SAStRE, M. y AGUILAR, E. (2003), Dirección de recursos humanos: un enfoque estratégico, McGraw-Hill, Madrid.

SERRANO, J. y BARBA, M. I. (2010), "La gestión de recursos humanos en las corporaciones locales", Cuadernos de Gestión, vol.12 (2): 149-168.

SHACK, N.; BARBOZA, M.; MENDOZA, J. y ReYES, O. (1996), "La Administración presupuestaria y su impacto en la Gestión Pública”, Magister en Gestión y Políticas Públicas, Universidad de Chile, Santiago de Chile.

SieGel, J. y SUmmermatTeR, L. (2008), "Defining Performance in Public Management: a Survey of Academic Journals", en Permanent Study Group on Performance in the Public Sector, European Group of Public Administration (EGPA), Annual Conference 2008, Rotterdam.

SOLE, F. y SCHIUMA, G. (2010), "Using Performance Measures in Public Organisations: Challenges of Italian Public Administrations", Measuring Business Excellence, vol. 14 (3): 70-84.

TORRES, L.; PINA, V. y MARTí, C. (2010), "Performance Measurement in Spanish Local Governments. An Empirical Study about the State of the Art", en $32^{\circ}$ European Group Of Public Administration (EGPA), Annual Conference Toulouse, France.

VAlle-CabreRA, R.J. (coord.) (2003), La gestión estratégica de los recursos humanos, Pearson-Prentice Hall, Madrid.

VILLORIA, M. y DEL PINO, E. (2009), Dirección y gestión de recursos humanos en las Administraciones Públicas, Editorial Tecnos, Madrid.

VILLORIA, M (2009), "La gestión de las personas en la Administración Pública. La evaluación del rendimiento", en Junta de Comunidades de Castilla-La Mancha, Consejería de Administraciones Públicas, Escuela de Administración Regional: Documentación sobre gerencia pública del subgrupo A1, Cuerpo Superior, especialidad de Administración General de la Administración de la Junta de Comunidades de Castilla-La Mancha.

VIVAS, S. y FERNÁNDEZ, R. (2011), "Gestión por competencias en instituciones sanitarias: claves de la dirección de recursos humanos en la Comunidad Valenciana (España)", Estudios Gerenciales, Vol. 27 (120): 41-61.

WALKER, R. y BOYNE, G. (2009), "Determinants of Performance in Public Organizations", Public Administration, Vol.87 (3): 433-439.

WARREN, L. y HUTCHINSON, W. (2000), "Success Factors for High-technology SMEs: A Case Study from Australia”, Journal of Small Business Management, Julio: 86-91.

WiLLIAMS, R. (2003), Rendimiento del personal, Thomson, Madrid.

YERGA, A. (2003), Estudio de los recursos humanos del sector público local en Andalucía, Consejería de Gobernación de la Junta de Andalucía.

YUSUF, A. (1995), "Critical Success Factors for Small Business: Perceptions of South Pacific Entrepreneurs", Journal of Small Business Management, April: 68-73. 